\title{
Comparative Statics for Size-Dependent Discounts in Matching Markets*
}

\author{
David Delacrétaz ${ }^{\dagger} \quad$ Scott Duke Kominers ${ }^{\ddagger} \quad$ Alexandru Nichifor ${ }^{\S}$
}

June 19, 2020

\begin{abstract}
We prove a natural comparative static for many-to-many matching markets in which agents' choice functions exhibit size-dependent discounts: reducing the extent to which some agent discounts additional partners leads to improved outcomes for the agents on the other side of the market, and worsened outcomes for the agents on the same side of the market. Our argument draws upon recently developed methods bringing tools from choice theory into matching.
\end{abstract}

JEL classification: C78, D47, D71

Keywords: Size-Dependent Discounts, Path-Independence, Respect for Improvements

${ }^{*}$ We thank Olivier Bochet, Simon Loertscher, Leslie Marx, and two anonymous referees for helpful comments and suggestions.

${ }^{\dagger}$ E-mail: david.delacretaz@economics.ox.ac.uk; Department of Economics and Nuffield College, University of Oxford, New Road, Oxford OX1 1NF, United Kingdom. Delacrétaz is grateful or the support of the University of Melbourne (a Visiting Research Scholar Award).

${ }^{\ddagger}$ E-mail: kominers@fas.harvard.edu; Entrepreneurial Management Unit, Harvard Business School, Arthur Rock Center for Entrepreneurship, Soldiers Field, Boston, MA 02163; Department of Economics and Center of Mathematical Sciences and Applications, Harvard University; and National Bureau of Economic Research, United States of America. Kominers is grateful for the support of the National Science Foundation (grant SES-1459912), the Harvard Center of Mathematical Sciences and Applications (the Ng Fund and the Mathematics in Economics Research Fund), and the University of Melbourne (the Centre for Market Design and an Eminent Research Scholar Award).

${ }^{\S}$ E-mail: nichifor@unimelb.edu.au; Faculty of Business and Economics, University of Melbourne, 111 Barry Street, Parkville, 3010, Victoria, Australia. Nichifor is grateful for the support of the Australian Research Council (Discovery Early Career Research Award DE170101183). 


\section{Introduction}

Delacrétaz et al. (2019) introduced a general family of valuation functions with sizedependent discounts, under which each agent's value for sets of potential partners is given by an additive valuation over individual agents, discounted by a term that depends on the total number of agents in the set. ${ }^{1}$ The additivity of $a$ 's valuations ensures that each agent with whom a may partner is evaluated independently from any other agent in the set; thus, there are no externalities among potential partners. Meanwhile, the discount term captures the idea that agent $a$ 's marginal value for partners decreases as his number of partners increases; thus, there is competition among potential partners.

We study many-to-many matching markets with size-dependent discounts and investigate the welfare implications of a discount reduction, under which an agent becomes more willing to accept additional trading partners. Our main result (Theorem 1) formally shows the intuitive comparative static that a decrease in one agent's discounts makes all other agents on his side of the market worse off, and all agents on the other side of the market better off. To prove our main result, we draw upon recently developed methods that bring tools from choice theory into matching: First, we show that valuation functions with size-dependent discounts induce path-independent choice functions; moreover, discount reductions lead to expansions of those choice functions. Then, to complete our proof, we invoke a powerful comparative static result of Chambers and Yenmez (2017) that applies to all expansions of path-independent choice functions. ${ }^{2}$ We also show that our main conclusion continues to hold if the discounts of all agents on one side of the market decrease (Corollary 1). However, the effect of a simultaneous reduction in the discounts of agents on opposite sides of the market is ambiguous, even if the discounts decrease by the exact same amount (Example 1). Finally, we show that our main findings can be sharpened to cover deferred-acceptance-like mechanisms by comparing side-optimal matchings (Corollary 2).

\section{Model}

There are finite sets of workers $W$ and firms $F$. The full set of agents is $I$, where $I=W \cup F$ (and $W \cap F=\emptyset$ ). We denote by $|A|$ the number of agents in $A \subseteq I$, and write $\mathcal{P}(I)=2^{I}$

\footnotetext{
${ }^{1}$ Relatedly, Farrell and Scotchmer (1988) model a situation in which the productivity of a group of agents depends on the number of agents in the group but not on their identities.

${ }^{2}$ As we discuss at the end of Section 3, for an alternative way to complete our proof, we could use the comparative static result of Pycia and Yenmez (2019) instead of that of Chambers and Yenmez (2017), as the two results are equivalent in our context.
} 
for the set of all subsets of agents.

For each agent $a \in I$, worker or firm, we let

$$
\bar{I}^{a} \equiv \begin{cases}F & a \in W \\ W & a \in F\end{cases}
$$

denote the set of agents on the other side of the market from $a$; we refer to the set of agents $I^{a}=I \backslash \bar{I}^{a}$ as being on the same side of the market as $a$.

For each agent $a \in I$, the valuation function $v^{a}: \bar{I}^{a} \rightarrow \mathbb{R}$ assigns a value to each individual agent on the other side of the market. We assume that each agent $a$ can withdraw from the market at some cost, which for simplicity we normalize to 0, i.e., $v^{a}(\emptyset)=0$.

We extend each agent $a$ 's valuation over individual agents to sets of agents by assuming size-dependent discounts: we require that there exists a vector of discounts $\delta^{a}=$ $\left(\delta_{1}^{a}, \ldots, \delta_{j}^{a}, \ldots \delta_{\left|\bar{I}^{a}\right|}^{a}\right)$, with $\delta_{1}^{a}=0, \delta_{j-1}^{a} \leq \delta_{j}^{a}$ for each $1<j \leq\left|\bar{I}^{a}\right|$, and such that for each $A \subseteq \bar{I}^{a}$

$$
v_{\delta}^{a}(A)=\sum_{x \in A} v^{a}(x)-\sum_{j=1}^{|A|} \delta_{j}^{a} \cdot 3
$$

We assume that each agent's valuation and discounts are such that the induced preferences over sets of agents on the other side of the market are strict. ${ }^{4}$

The lists of size-dependent discounts $\delta^{a}$ and valuations $v_{\delta}^{a}$ for each agent $a$ give rise to the discount profile $\delta=\left(\delta^{a}\right)_{a \in I}$ and associated valuation profile $v_{\delta}^{I}=\left(v_{\delta}^{a}\right)_{a \in I}$.

For each agent $a \in I$, and each $A \subseteq \bar{I}^{a}$, the choice function induced by a's valuation selects that agent's most preferred set of other-side agents, i.e.,

$$
C_{\delta}^{a}(A)=\underset{A^{\prime} \subseteq A}{\arg \max }\left\{v_{\delta}^{a}\left(A^{\prime}\right)\right\}
$$

Note that since the induced preferences are strict, the set of agents selected by the choice function $C_{\delta}^{a}$ is unique, so we may think of $C_{\delta}^{a}(A)$ as a set of agents.

A (many-to-many) matching $\mu$ assigns to each agent $a$ a set of agents on the other side of the market $\bar{I}^{a}$, allowing for the possibility that a remains unmatched. That is, $\mu(a) \in \mathcal{P}\left(\bar{I}^{a}\right)$ and $b \in \mu(a)$ if and only if $a \in \mu(b)$. A matching $\mu$ is individually rational if no agent $a$ prefers to unilaterally drop some of the agents with whom he is matched at $\mu(a)$, i.e., if

\footnotetext{
${ }^{3}$ For simplicity, with some abuse of notation, we write $v_{\delta}^{a}$ instead of $v_{\delta^{a}}^{a}$.

${ }^{4}$ Our size-dependent discounts condition corresponds to the "one block" non-monotonic version of Delacrétaz et al. (2019).
} 
for each $a \in I$, we have $C_{\delta}^{a}(\mu(a))=\mu(a)$. A matching $\mu$ is unblocked if there does not exist a nonempty set of worker-firm pairs $X \subseteq W \times F$ such that, for every $a \in I$, letting $\bar{X}^{a} \equiv\left\{b \in \bar{I}^{a}:\{a, b\} \in X\right\}$, we have $\bar{X}^{a} \cap \mu(a)=\emptyset$ and $\bar{X}^{a} \subseteq C_{\delta}^{a}\left(\mu(a) \cup \bar{X}^{a}\right)$. A matching $\mu$ is stable if it is both individually rational and unblocked. A matching $\mu$ is (revealed) preferred to a matching $\mu^{\prime}$ by agent $a$ if $C_{\delta}^{a}\left(\mu(a) \cup \mu^{\prime}(a)\right)=\mu(a)$.

\section{$2.1 \quad$ Illustration}

Valuations with size-dependent discounts naturally expand the class of preferences that agents are usually allowed to express in two-sided matching markets with capacity constraints. ${ }^{5}$ Indeed, in prior two-sided models of matching with capacity constraints, the constraints are typically "hard," in the sense that they are fixed irrespective of which partners are available. ${ }^{6}$ Moreover, those prior models often assume a coarse form for the language an agent may use to express its preferences over partners - such as requiring a single rank-order list (with the resulting preferences being responsive; see Roth (1985)). Under size-dependent discount valuations, by contrast, the number of available positions (and associated cut-offs) can be more flexible.

For instance, in a school choice setting, we might think of a school as being able to accommodate anywhere between $N-\underline{n}$ and $N+\bar{n}$ students (with $\underline{n}$ not necessarily equal to $\bar{n}$ ), where the number of admitted students is more finely calibrated via a series of increasing size-dependent discount factors $\delta_{N-\underline{n}} \leq \ldots \leq \delta_{N} \leq \ldots \leq \delta_{N+\bar{n}}$. The interpretation is that the school's maximal physical capacity is $N+\bar{n}$; and "on average" a group of $N$ students should be admitted, but only if all of their application scores are above $\delta_{N}$; moreover, the number of available positions flexes upwards and downwards as a function of the quality of the applicants. ${ }^{7}$ Note that the ability to express "soft" capacity constraints may help open up the possibility of achieving fair and non-wasteful assignments of students to schools (Ehlers et al., 2014). ${ }^{8}$

\footnotetext{
${ }^{5}$ Valuations with size-dependent discounts also appear in auction/procurement settings (Delacrétaz et al., 2019).

${ }^{6}$ For a recent survey of the matching with constraints literature, see Kamada and Kojima (2017).

${ }^{7}$ To embed hard constraints, such as a school's maximal physical capacity $N+\bar{n}$, it suffices to set the discount term $\delta_{N+\bar{n}+1}$ to a very high value (in this case, larger than the highest possible score).

${ }^{8}$ The general family of valuation functions with size-dependent discounts of Delacrétaz et al. (2019) allows for various "blocks" of objects, where each block corresponds to, e.g., a different commodity. Ehlers et al. (2014) allowed for various student types that may be determined by, e.g., ethnicity or some other socioeconomic criteria. Here, for simplicity, we consider the one-block version of the size-dependent discounts valuation of Delacrétaz et al. (2019), corresponding to same types in the setting of Ehlers et al. (2014).
} 


\section{Results}

Our main result shows that under stable matching, reducing some agent's discounts leads to worse outcomes for other agents on the same side of the market, and better outcomes for agents on the other side of the market. Formally, for an agent $a$ with discounts $\delta^{a}$, a discount reduction occurs when we decrease some subset of the agent's discount values, i.e., we replace $a^{\prime}$ s discounts $\delta^{a}$ with $\varepsilon^{a} \equiv\left(\varepsilon_{1}^{a}, \ldots, \varepsilon_{k}^{a}, \ldots \varepsilon_{\left|\bar{I}^{a}\right|}^{a}\right)$ such that $\varepsilon_{k}^{a} \leq \delta_{k}^{a}$ for each $1 \leq k \leq\left|\bar{I}^{a}\right|$ (and $\left.\varepsilon_{1}^{a}=\delta_{1}^{a}=0\right) .{ }^{9}$ We use the standard notation $v_{\delta}^{-a}=v_{\delta}^{I \backslash\{a\}}$, and denote by $\left(v_{\varepsilon}^{a}, v_{\delta}^{-a}\right)$ the "post-discount-reduction" valuation profile obtained from $v_{\delta}^{I}$ by replacing $v_{\delta}^{a}$ with $v_{\varepsilon}^{a}$.

Our main result is then as follows.

Theorem 1. Consider any profile of size-dependent discounts $\delta$ with associated valuation profile $v_{\delta}^{I}$. Fix any agent $a \in I$, and consider any post-discount-reduction profile $\left(v_{\varepsilon}^{a}, v_{\delta}^{-a}\right)$. For every matching $\mu$ that is stable under $v_{\delta}^{I}$, there exists a matching $\mu^{\prime}$ stable under $\left(v_{\varepsilon}^{a}, v_{\delta}^{-a}\right)$ such that:

1. $\mu^{\prime}$ is preferred to $\mu$ by all agents on the other side of the market from a; and,

2. $\mu$ is preferred to $\mu^{\prime}$ by all agents (other than a) on the same side of the market as a.

To prove Theorem 1, we appeal to a series of powerful comparative static results that Chambers and Yenmez (2017) proved for matching markets in which all agents have pathindependent choice functions. We first show that if agents' valuations exhibit size-dependent discounts, then: (i) the induced choice functions are path-independent; (ii) following a weak decrease in one of the discounts of an agent $a$, the resulting choice function is an expansion of $a$ 's initial choice function. Together with results of Chambers and Yenmez (2017), (i) and (ii) allow us to prove Theorem 1.

\section{Size-Dependent Discounts Induce Path-Independent Choice Functions}

To show that size-dependent discounts induce path-independent choice functions, we show that if agents' valuations exhibit size-dependent discounts, then the agents' choice functions satisfy the well-known irrelevance of rejected agents and substitutability conditions (Lemmata 1 and 2), which together imply path-independence (Lemma 3).

\footnotetext{
${ }^{9}$ Our requirement that $\varepsilon_{1}^{a}=\delta_{1}^{a}=0$ is natural because it means that both $v_{\delta}^{a}$ and $v_{\varepsilon}^{a}$ correspond to the same valuations $v^{a}$ over individual agents in $\bar{I}^{a}$.
} 
First, we note that valuations exhibiting size-dependent discounts induce choice functions that satisfy the irrelevance of rejected agents condition, which requires that an agent $a$ 's choice is unaffected by the removal of a set of agents that $a$ was not choosing.

Definition 1. An agent $a$ 's choice function $C_{\delta}^{a}$ satisfies the irrelevance of rejected agents condition if for all $A^{\prime}, A \subseteq \bar{I}^{a}$ such that $C_{\delta}^{a}(A) \subseteq A^{\prime} \subseteq A$, we have $C_{\delta}^{a}(A)=C_{\delta}^{a}\left(A^{\prime}\right){ }^{10}$

Lemma 1. If an agent's valuation exhibits size-dependent discounts, then his choice function satisfies the irrelevance of rejected agents condition.

Proof. Suppose to the contrary that $C_{\delta}^{a}\left(A^{\prime}\right) \neq C_{\delta}^{a}(A)$ : then, since $a^{\prime}$ 's induced preferences are strict, we must have $v_{\delta}^{a}\left(C_{\delta}^{a}(A)\right) \neq v_{\delta}^{a}\left(C_{\delta}^{a}\left(A^{\prime}\right)\right)$. Then, the fact that $C_{\delta}^{a}(A) \subseteq A^{\prime}$ implies that $v_{\delta}^{a}\left(C_{\delta}^{a}(A)\right)<v_{\delta}^{a}\left(C_{\delta}^{a}\left(A^{\prime}\right)\right)$ (because $C_{\delta}^{a}(A)$ can be chosen but is not optimal when the agents in $A^{\prime}$ are available). However, the fact that $C_{\delta}^{a}\left(A^{\prime}\right) \subseteq A^{\prime} \subseteq A$ implies that $v_{\delta}^{a}\left(C_{\delta}^{a}(A)\right)>$ $v_{\delta}^{a}\left(C_{\delta}^{a}\left(A^{\prime}\right)\right)$ (because $C_{\delta}^{a}\left(A^{\prime}\right)$ can be chosen but is not optimal when the agents in $A$ are available), a contradiction.

Next, we note that valuations exhibiting size-dependent discounts induce choice functions that are substitutable in the sense that whenever the set of agents available to choose from shrinks, all agents who were initially chosen and are still available remain chosen.

Definition 2. An agent $a$ 's choice function is substitutable if for any distinct $A^{\prime}, A \subseteq \bar{I}^{a}$, we have that $C_{\delta}^{a}\left(A^{\prime} \cup A\right)=A^{\prime}$ implies $a^{\prime} \in C_{\delta}^{a}\left(\left\{a^{\prime}\right\} \cup A\right)$ for any $a^{\prime} \in A^{\prime}{ }^{11}$

Lemma 2 (Delacrétaz et al. (2020)). If an agent's valuation exhibits size-dependent discounts, then his choice function is substitutable.

Finally, a choice function is path-independent if, when the set of agents available to choose from is partitioned into two subsets, the choice from the initial set of agents coincides with the choice over the independent choices from each of the sets in the partition.

Definition 3. An agent $a$ 's choice function $C_{\delta}^{a}$ is path-independent if for every $A^{\prime}, A \subseteq \bar{I}^{a}$, we have $C_{\delta}^{a}\left(A^{\prime} \cup A\right)=C_{\delta}^{a}\left(C_{\delta}^{a}\left(A^{\prime}\right) \cup C_{\delta}^{a}(A)\right){ }^{12}$

\footnotetext{
${ }^{10}$ Blair (1988), Alkan and Gale (2003), and Chambers and Yenmez (2017) use the term "consistency" instead of irrelevance of rejected agents (Aygün and Sönmez, 2013); we adopt the later terminology to avoid any potential confusion with consistency as a normative requirement (see, e.g., Thomson (ming)).

${ }^{11}$ Substitutability is essential for establishing the existence of stable outcomes in various two-sided matching settings (see, e.g., Kelso and Crawford (1982) and Roth (1984)); moreover, under substitutability, as shown by Hatfield and Milgrom (2005), simple deferred-acceptance-like auctions can be used to find a solution. Beyond two-sided settings, (full) substitutability remains an essential condition (Hatfield et al., 2013, 2019, ming); for a history of substitutability, see Hatfield et al. (2019).

${ }^{12}$ Path-independence, first formally introduced by Plott (1973), is a key property in the choice-theory literature; for a survey, see Moulin (1985).
} 
By Lemma 1 of Chambers and Yenmez (2017), choice functions are path-independent if (and only if) they satisfy the independence of rejected agents and substitutability conditions ${ }^{13}$ hence, the following result is immediate from our Lemmata 1 and 2.

Lemma 3. If an agent's valuation exhibits size-dependent discounts, then his choice function is path-independent.

\section{Choice Expansion under Size-Dependent Discounts}

Now, we prove Theorem 1 by way of comparative static results of Chambers and Yenmez (2017) for path-independent choice functions. We first show by induction that if an agent $a$ 's valuation exhibits size-dependent discounts, then a discount reduction from $\delta^{a}$ to $\varepsilon^{a}$ induces a new choice function $C_{\varepsilon}^{a}$ that is an expansion of $C_{\delta}^{a}$, in the sense that for any set of agents on the other side of the market, every agent chosen under $C_{\delta}^{a}$ is also chosen under $C_{\varepsilon}^{a}$.

Definition 4. An agent $a$ 's choice function $\widehat{C}^{a}$ is an expansion of $C_{\delta}^{a}$ if for every $A \subseteq \bar{I}^{a}$, we have $\widehat{C}^{a}(A) \supseteq C_{\delta}^{a}(A){ }^{14}$

Lemma 4. If an agent's valuation exhibits size-dependent discounts, then a discount reduction for that agent leads to a choice function that is an expansion of the agent's original choice function.

Proof. First, we fix an agent $a$, a set of agents $A \subseteq \bar{I}^{a}$, and a discount profile $\delta$. Without loss of generality, we index the agents in $A$ in decreasing order of their individual values to $a: A=\left\{b_{1}, b_{2}, \ldots, b_{|A|}\right\}$ with $v^{a}\left(b_{1}\right)>v^{a}\left(b_{2}\right)>\ldots>v^{a}\left(b_{|A|}\right){ }^{15}$

Let $\ell \equiv\left|C_{\delta}^{a}(A)\right|$ be the number of agents that $a$ chooses. We show that $C_{\delta}^{a}(A)=$ $\left\{b_{1}, \ldots, b_{\ell}\right\}$. Suppose to the contrary that $C_{\delta}^{a}(A) \neq\left\{b_{1}, \ldots, b_{\ell}\right\}$. Then, there exist $n \leq \ell$ such that $b_{n} \notin C_{\delta}^{a}(A)$ and $m>\ell$ such that $b_{m} \in C_{\delta}^{a}(A)$, so

$$
v_{\delta}^{a}\left(\left(C_{\delta}^{a}(A) \cup\left\{b_{n}\right\}\right) \backslash\left\{b_{m}\right\}\right)=v_{\delta}^{a}\left(C_{\delta}^{a}(A)\right)+v^{a}\left(b_{n}\right)-v^{a}\left(b_{m}\right) .
$$

As $n<m, v^{a}\left(b_{n}\right)>v^{a}\left(b_{m}\right)$. Therefore, $v_{\delta}^{a}\left(\left(C_{\delta}^{a}(A) \cup\left\{b_{n}\right\}\right) \backslash\left\{b_{m}\right\}\right)>v_{\delta}^{a}\left(C_{\delta}^{a}(A)\right)$, which contradicts the assumption that $C_{\delta}^{a}(A)$ is the subset of $A$ that maximizes $v_{\delta}^{a}$.

\footnotetext{
${ }^{13}$ This result was initially stated in a different setting and without proof by Aizerman and Malishevski (1981, Corollary 2); that version of the result was then formally shown to be correct by Moulin (1985, Lemma 6).

${ }^{14}$ Chambers and Yenmez (2017) list a number of practical situations in which choice function expansions play an important role, such as in controlled school choice (Hafalir et al., 2013; Ehlers et al., 2014) and residency matching (Kamada and Kojima, 2015).

${ }^{15}$ As the induced preferences are strict, agent $a$ does not have the same value for any two agents in $A$.
} 
Now, we let $\varepsilon$ be a discount profile such that $\varepsilon \leq \delta$. To prove the lemma, we need to show that $C_{\delta}^{a}(A) \subseteq C_{\varepsilon}^{a}(A)$. We have shown that $C_{\delta}^{a}(A)=\left\{b_{1}, \ldots, b_{\ell}\right\}$.

Letting $\ell^{\prime} \equiv\left|C_{\varepsilon}^{a}(A)\right|$, analogous reasoning shows that $C_{\varepsilon}^{a}(A)=\left\{b_{1}, \ldots, b_{\ell^{\prime}}\right\}$; therefore, we need to show that $\ell^{\prime} \geq \ell$. Suppose to the contrary that

$$
\ell^{\prime}<\ell
$$

In that case, we have

$$
v^{a}\left(b_{\ell^{\prime}+1}\right)<\varepsilon_{\ell^{\prime}+1}^{a},
$$

as otherwise we would have

$$
v_{\varepsilon}^{a}\left(C_{\varepsilon}^{a}(A) \cup\left\{b_{\ell^{\prime}+1}\right\}\right)=v_{\varepsilon}^{a}\left(C_{\varepsilon}^{a}(A)\right)+v^{a}\left(b_{\ell^{\prime}+1}\right)-\varepsilon_{\ell^{\prime}+1}^{a}>v_{\varepsilon}^{a}\left(C_{\varepsilon}^{a}(A)\right),
$$

contradicting the fact that $C_{\varepsilon}^{a}(A)$ is the subset of $A$ that maximizes $v_{\varepsilon}^{a}$.

Our hypothesis (2) implies that

$$
\ell^{\prime}+1 \leq \ell
$$

hence, as $\varepsilon \leq \delta$ and $\delta_{1}^{a} \leq \delta_{2}^{a} \leq \ldots \leq \delta_{\left|\bar{I}^{a}\right|}^{a}$, (3) implies that

$$
\delta_{\ell}^{a} \stackrel{b_{\ell} \in C_{\delta}^{a}(A)}{\leq} v^{a}\left(b_{\ell}\right) \stackrel{(4)}{\leq} v^{a}\left(b_{\ell^{\prime}+1}\right) \stackrel{(3)}{<} \varepsilon_{\ell^{\prime}+1}^{a} \stackrel{(\varepsilon \leq \delta)}{\leq} \delta_{\ell^{\prime}+1}^{a} \stackrel{(4)}{\leq} \delta_{\ell}^{a}
$$

a contradiction.

Given Lemmata 3 and 4, Theorem 1 follows immediately from Theorem 2 of Chambers and Yenmez (2017), which shows that an expansion induces the desired comparative static so long as all agents' choice functions are path-independent. Alternatively, given Lemmata 1, 2, and 4, Theorem 1 also follows immediately from Theorem 7 of Pycia and Yenmez (2019). ${ }^{16}$

\section{Discussion}

We can apply our Theorem 1 iteratively to show that following a discount reduction for every agent on one side of the market, all agents on the other side become better off - a result that

\footnotetext{
${ }^{16}$ To see this, note that Pycia and Yenmez (2019) consider a more general matching model with externalities, and thus have to formulate both their irrelevance of rejected contracts and substitutability conditions with respect to a reference set; in the absence of externalities, their model and conditions coincide with ours, and their comparative static result is equivalent to that of Chambers and Yenmez (2017).
} 
mirrors Corollary 2 of Chambers and Yenmez (2017).

Corollary 1. Suppose that all agents' valuations exhibit size-dependent discounts and consider any profile of size-dependent discounts $\delta$ with associated valuation profile $v_{\delta}^{I}$. For each firm $f \in F$, consider a discount reduction from $\delta^{f}$ to $\varepsilon^{f}$ and denote the post-discountreduction valuation profile by $\left(v_{\varepsilon}^{F}, v_{\delta}^{W}\right)$. Then, for any matching $\mu$ that is stable under $v_{\delta}^{I}$, there exists a matching $\mu^{\prime}$ that is stable under $\left(v_{\varepsilon}^{F}, v_{\delta}^{W}\right)$ such that every worker $w$ prefers $\mu^{\prime}$ to $\mu$.

By combining our results in Theorem 1 with Corollary 1 of Chambers and Yenmez (2017), we obtain a sharpening of our main result that speaks to deferred-acceptance-like mechanisms by comparing side-optimal stable matchings. ${ }^{17}$ Formally, we say that a stable matching $\mu$ is firm-optimal if each firm prefers it to any other stable matching $\mu^{\prime}$. Likewise, a stable matching $\mu$ is worker-optimal if each worker prefers it to any other stable matching $\mu^{\prime}$.

Corollary 2. Suppose that all agents' valuations exhibit size-dependent discounts and consider any profile of size-dependent discounts $\delta$ with associated valuation profile $v_{\delta}^{I}$. Fix any agent $f \in F$, and consider any post-discount-reduction profile $\left(v_{\varepsilon}^{f}, v_{\delta}^{-f}\right)$. Let $\mu^{F}$ be the firmoptimal and $\mu^{W}$ be the worker-optimal stable matchings under $v_{\delta}^{I}$. Let $\bar{\mu}^{F}$ be the firm-optimal and $\bar{\mu}^{W}$ be the worker-optimal stable matchings under $\left(v_{\varepsilon}^{f}, v_{\delta}^{-f}\right) .{ }^{18}$ Then, the following hold:

1. every worker prefers $\bar{\mu}^{W}$ to $\mu^{W}$, and every firm other than $f$ prefers $\mu^{W}$ to $\bar{\mu}^{W}$; and,

2. every worker prefers $\bar{\mu}^{F}$ to $\mu^{F}$, and every firm other than $f$ prefers $\mu^{F}$ to $\bar{\mu}^{F}$.

It is clear from our argument for Theorem 1 that the result extends to settings in which agents other than the one undergoing a discount reduction have general path-independent choice functions - it is not necessary that all agents' preferences exhibit size-dependent discounts.

Moreover, Theorem 1 is "sharp" in the sense that a simultaneous discount reduction for agents on different sides of the market may have an ambiguous effect. Indeed, suppose that all agents' valuations exhibit size-dependent discounts and consider any profile of size-dependent discounts $\delta$ with associated valuation profile $v_{\delta}^{I}$. We consider simultaneous discount reductions on both sides of the market fixing, say, $f \in F$ and $w \in W$ and taking discount reductions from $\delta^{f}$ to $\varepsilon^{f}$ and from $\delta^{w}$ to $\varepsilon^{w}$. As the following example illustrates, with discount reductions on both sides, the conclusion of Theorem 1 may not hold.

\footnotetext{
${ }^{17}$ For a description of a many-to-many deferred acceptance mechanism, see Chambers and Yenmez (2017).

${ }^{18}$ These extremal stable matchings exist because size-dependent discounts induce substitutable choice functions (Lemma 2).
} 
Example 1. Let $F=\left\{f_{1}, f_{2}, f_{3}\right\}, W=\left\{w_{1}, w_{2}, w_{3}\right\}$, and $I=F \cup W$. The valuations over individual agents are as follows:

\begin{tabular}{|lll||lll|}
\hline$v^{f_{1}}\left(w_{1}\right)=1$ & $v^{f_{2}}\left(w_{1}\right)=-1$ & $v^{f_{3}}\left(w_{1}\right)=1$ & $v^{w_{1}}\left(f_{1}\right)=1$ & $v^{w_{2}}\left(f_{1}\right)=1$ & $v^{w_{3}}\left(f_{1}\right)=3$ \\
$v^{f_{1}}\left(w_{2}\right)=3$ & $v^{f_{2}}\left(w_{2}\right)=3$ & $v^{f_{3}}\left(w_{2}\right)=3$ & $v^{w_{1}}\left(f_{2}\right)=-1$ & $v^{w_{2}}\left(f_{2}\right)=3$ & $v^{w_{3}}\left(f_{2}\right)=1$ \\
$v^{f_{1}}\left(w_{3}\right)=5$ & $v^{f_{2}}\left(w_{3}\right)=5$ & $v^{f_{3}}\left(w_{3}\right)=5$ & $v^{w_{1}}\left(f_{3}\right)=5$ & $v^{w_{2}}\left(f_{3}\right)=5$ & $v^{w_{3}}\left(f_{3}\right)=5$ \\
\hline
\end{tabular}

For each agent $a \in I$, let the vector of discounts be $\delta^{a}=(0,9,10)$ and the associated valuation over sets of agents be $v_{\delta}^{a}$. Recall that valuations over sets of agents are determined by using the discount vector to extend the valuations over individual agents (see equation 1 ); for example, the value of agent $w_{1}$ for being matched to both $f_{1}$ and $f_{3}$ is

$$
v_{\delta}^{w_{1}}\left(\left\{f_{1}, f_{3}\right\}\right)=v^{w_{1}}\left(f_{1}\right)+v^{w_{1}}\left(f_{3}\right)-\delta_{2}^{w_{1}}=1+5-9=-3
$$

For the profile of size-dependent discounts $\delta=\left(\delta^{a}\right)_{a \in I}$ with associated valuation profile $v_{\delta}^{I}=\left(v_{\delta}^{a}\right)_{a \in I}$, there is a unique stable matching $\mu$, such that

$$
\mu\left(f_{1}\right)=w_{1}, \mu\left(f_{2}\right)=w_{2}, \mu\left(f_{3}\right)=w_{3} \cdot{ }^{19}
$$

We now consider the effects of a simultaneous (and identical) discount reduction for $f_{3}$ and $w_{3}$; the discounts for all other agents are left unchanged. Let $\varepsilon^{f_{3}}=\varepsilon^{w_{3}}=(0,2,10)$, and for each $a \in I \backslash\left\{f_{3}, w_{3}\right\}$, let $\varepsilon^{a}=\delta^{a}$. For the profile of size-dependent discounts $\varepsilon=\left(\varepsilon^{a}\right)_{a \in I}$ with associated valuation profile $v_{\varepsilon}^{I}=\left(v_{\varepsilon}^{a}\right)_{a \in I}$, there is a unique stable matching $\mu^{\prime}$, such that

$$
\mu^{\prime}\left(f_{1}\right)=w_{3}, \mu^{\prime}\left(f_{3}\right)=\left\{w_{2}, w_{3}\right\}, \mu^{\prime}\left(w_{3}\right)=\left\{f_{1}, f_{3}\right\}
$$

under which agents $f_{2}$ and $w_{1}$ are unmatched.

Thus, matching $\mu^{\prime}$ is preferred to $\mu$ by agents $f_{1}, f_{3}, w_{2}, w_{3}$, while matching $\mu$ is preferred to $\mu^{\prime}$ by agents $f_{2}$ and $w_{1}$.

\footnotetext{
${ }^{19}$ To see that $\mu$ is the unique stable matching, note that: (i) the discounts are so large that each agent chooses at most one partner; (ii) the valuations over individual agents induce a positive assortative matching in which the worker and firm with the first, second, and third highest value for each other are matched.
} 


\section{References}

Aizerman, M. and A. Malishevski (1981). General theory of best variants choice: Some aspects. IEEE Transactions on Automatic Control 26(5), 1030-1040.

Alkan, A. and D. Gale (2003). Stable schedule matching under revealed preference. Journal of Economic Theory 112, 289-306.

Aygün, O. and T. Sönmez (2013). Matching with contracts: Comment. American Economic Review 103(5), 2050-2051.

Blair, C. (1988). The lattice structure of the set of stable matchings with multiple partners. Mathematics of Operations Research 13, 619-628.

Chambers, C. P. and M. B. Yenmez (2017). Choice and matching. American Economic Journal: Microeconomics 9, 126-147.

Delacrétaz, D., S. D. Kominers, and A. Nichifor (2020). Size-dependent discounts for matching markets and auctions. Working paper.

Delacrétaz, D., S. Loertscher, L. M. Marx, and T. Wilkening (2019). Two-sided allocation problems, decomposability, and the impossibility of efficient trade. Journal of Economic Theory 179, 416-454.

Ehlers, L., I. E. Hafalir, M. B. Yenmez, and M. A. Yildirim (2014). School choice with controlled choice constraints: Hard bounds versus soft bounds. Journal of Economic Theory 153, 648-683.

Farrell, J. and S. Scotchmer (1988). Partnerships. The Quarterly Journal of Economics 103(2), 279-297.

Hafalir, I. E., M. B. Yenmez, and M. A. Yildirim (2013). Effective affirmative action in school choice. Theoretical Economics 8, 325-363.

Hatfield, J. W., S. D. Kominers, A. Nichifor, M. Ostrovsky, and A. Westkamp (2013). Stability and competitive equilibrium in trading networks. Journal of Political Economy 121(5), 966-1005.

Hatfield, J. W., S. D. Kominers, A. Nichifor, M. Ostrovsky, and A. Westkamp (2019). Full substitutability. Theoretical Economics 14(4), 1535-1590. 
Hatfield, J. W., S. D. Kominers, A. Nichifor, M. Ostrovsky, and A. Westkamp (forthcoming). Full substitutability. Theoretical Economics.

Hatfield, J. W. and P. R. Milgrom (2005). Matching with contracts. American Economic Review 95, 913-935.

Kamada, Y. and F. Kojima (2015). Efficient matching under distributional constraints: Theory and applications. American Economic Review 105, 67-99.

Kamada, Y. and F. Kojima (2017). Recent developments in matching with constraints. American Economic Review Papers $\& 3$ Proceedings 107(5), 200-204.

Kelso, A. S. and V. P. Crawford (1982). Job matching, coalition formation, and gross substitutes. Econometrica 50, 1483-1504.

Moulin, H. (1985). Choice functions over a finite set: a summary. Social Choice and Welfare $2(2), 147-160$.

Plott, C. R. (1973). Path independence, rationality, and social choice. Econometrica, 10751091.

Pycia, M. and M. B. Yenmez (2019). Matching with externalities. CEPR Discussion Paper No. DP13994. Available at https://ssrn.com/abstract=3464522.

Roth, A. E. (1984). Stability and polarization of interests in job matching. Econometrica 52, $47-57$.

Roth, A. E. (1985). The college admissions problem is not equivalent to the marriage problem. Journal of Economic Theory 36, 277-288.

Thomson, W. (forthcoming). Consistent Allocation Rules. Cambridge University Press. 